\title{
Evaluating the Performance of DEEC Variants
}

\author{
Rajeev Kumar \\ Department of Information Technology \\ DAV Institute of Engineering \& Technology \\ Jalandhar, Punjab, 144003, India
}

\author{
Rajdeep Kaur \\ Department of Computer Science \& Engineering \\ DAV Institute of Engineering \& Technology \\ Jalandhar, Punjab, 144003, India
}

\begin{abstract}
Wireless Sensor Networks (WSNs) have many sensor nodes having restricted battery power, which transmit sensed data to the Base Station that needs high energy consumption. Numerous routing protocols have been proposed in this regard getting energy efficiency in heterogeneous situations. Though, each protocol is inappropriate for heterogeneous WSNs. Efficiency of WSNs declines as varying the heterogeneity of sensor nodes. This paper has evaluated the performance of various Distributed Energy- Efficient Clustering based protocols like DEEC, DDEEC, EDEEC, HDEEC, EDDEEC and TDEEC under numerous scenarios; comprising various level of heterogeneity. MATLAB tool is used for experimental purpose. The comparison has shown that the EDDEEC has very effective results over other DEEC variants due to its special feature of T-absolute i.e. it treats all heterogeneous sensor nodes with same election probability when each node has lesser energy than T-absolute.
\end{abstract}

\section{Keywords}

WSNs, DEEC, heterogeneity, super nodes, T-absolute

\section{INTRODUCTION}

Wireless sensor networks consist of small nodes with sensing, computation, and wireless communication capabilities. Many routing, power management, and data dissemination protocols have been specifically designed for WSNs where energy awareness is an essential design issue. As wireless sensor network is a collection of sensor nodes therefore these sensor nodes are grouped into clusters. Each cluster is assigned with a leader, which is also called the cluster head $(\mathrm{CH})$ and usually performs the unique tasks like data aggregation, pass data to base station.

Various clustering protocols are specified in this consideration $[2,3]$. Every node belonging to cluster transmits their data to Cluster Head, where, Cluster Head performs the data aggregation and transmit this aggregated data to base station. In aggregation process, smaller numbers of messages are transmitted to Base Station and only some nodes have to send data to large distances therefore more energy is saved and the network lifetime is spanned. The cluster arrangement process is a two-level hierarchy process where the $\mathrm{CH}$ nodes make the higher level and the cluster-member nodes make the lower level. The sensor nodes at regular intervals send their data to the corresponding $\mathrm{CH}$ nodes. $\mathrm{CH}$ nodes aggregate the data and transmit them to the base station (BS) directly or through the midway communication with other $\mathrm{CH}$ nodes. Due to the $\mathrm{CH}$ nodes transmit all the time data to large distances than the member nodes; they obviously spend energy at large rates. A general solution in order to balance the energy consumption amongst all the sensor nodes is to regularly re-elect new CHs in each cluster. Low-Energy Adaptive Clustering Hierarchy (LEACH) [5], Power Efficient Gathering in Sensor Information Systems (PEGASIS) [7], Hybrid Energy-Efficient Distributed clustering (HEED) [8] are algorithms specially designed for homogenous WSN under thought because these protocols do not work effectively in heterogeneous circumstances as these algorithms are not capable to treat nodes in a different way in conditions of their energy. Whereas, Stable Election Protocol (SEP) [9], Distributed Energy-Efficient Clustering (DEEC) [10], Developed DEEC (DDEEC) [11], Enhanced DEEC (EDEEC) [12] and Threshold DEEC (TDEEC) [13] are algorithms designed for heterogeneous WSN.

In this paper, we study performance of heterogeneous WSNs protocols under three and multi-level heterogeneous networks. We have compared the performance of DEEC, DDEEC, EDEEC, TDEEC, EDDEEC, and HDEEC for different scenarios of three and multilevel heterogeneous WSNs. Heterogeneous WSNs contains normal, advanced and super sensor nodes where super nodes comes up with extra energy than to normal and advanced sensor nodes. We recognize each protocol focused around the extending stability phase, network life time of network alive through out rounds for a few three level heterogeneous networks. Each node having apparently equivalent proportion of normal, advanced and super nodes alongside the multilevel heterogeneous WSNs. It is observed that diverse protocols have distinctive effectiveness for three level and multilevel heterogeneous WSNs in terms of stability period, nodes alive and network life time. DEEC and DDEEC perform well under three level heterogeneous WSNs holding high energy level distinction between normal, advanced and super nodes regarding stability period. However, it is deficient in results as contrast with EDEEC and TDEEC.

The rest of the paper is ordered as follows: section 2 briefly reviews the related work. In section 3 , describes the network model. In section 4, radio dissipation model is explained. Proposed overview of Heterogeneous Protocols explained in section 5. In section 6 , we define the performance criteria. Section 7 shows the performance of different DEEC protocols by simulations and comparison. Finally, section 8 gives conclusion and future work.

\section{RELATED WORK}

W.R. Heinzelman, et al. [5] proposed a LEACH clustering algorithm for homogeneous WSNs in which nodes randomly choose among them to be $\mathrm{CHs}$ and transmit this choice criterion over the whole network to issue energy load. G. Smaragdakis, et al. [9] introduced SEP protocol in which each sensor node in a heterogeneous two level hierarchical network autonomously chooses itself as a $\mathrm{CH}$ on the basis of its initial energy relative to the other nodes of the network. L .Qing, Q. Zhu and M. Wang [10] introduced a protocol DEEC in which $\mathrm{CH}$ election is based on the probability of the ratio of residual energy and average energy of the network. Brahim Elbhiri, et al. [11] introduced a protocol DDEEC on the basis of residual energy for $\mathrm{CH}$ election to stable it over the whole network. So, the advanced nodes are more likely to be elected as $\mathrm{CH}$ for the first transmission rounds, and when their energy decreases, these nodes will have the same $\mathrm{CH}$ election probability like the 
normal nodes. P. Saini et al. [12] proposed a protocol EDEEC which is extended up to three level heterogeneity by adding an extra amount of energy level known as super nodes. Parul Saini and Ajay K Sharma [13] proposed a protocol TDEEC scheme selects the $\mathrm{CH}$ from the high energy nodes improving energy efficiency and lifetime of the network.Ease of Use. M.Y. Khan et al. [14] proposed a protocol called H-DEEC In H-DEEC, the chosen Cluster Heads (CHs) communicate the Base Station (BS) through beta elected nodes, by using multi-hopping. Logically divide the network into two parts, on the basis of the residual energy of nodes. The normal nodes with high initial and residual energy will be highly probable to be $\mathrm{CHs}$ than the nodes with lesser energy. N. Javaid et al. [15] have proposed protocol EDDEEC implements the idea of probabilities for $\mathrm{CH}$ selection based on initial, remaining energy level of the nodes and average energy of network.

\section{HETEROGENEOUS WSN MODEL}

In this segment, we suppose $\mathrm{N}$ number of nodes distributed in a square area of dimension M x M. Heterogeneous WSNs have two, three or multi types of nodes which are having different energy levels and are considered as two, three and multi-level heterogeneous WSNs respectively.

\subsection{Two Level Heterogeneous WSNs Model}

In two level heterogeneity, Eo describes the normal node's energy value and $E o(1+a)$ describes the advanced node's energy value where $a$ defines the value that is this amount of value times more energy contained by advanced node compared to the normal nodes. If two level heterogeneous network $N$ is the total number of nodes where $\mathrm{Nm}$ defines total number of advanced nodes and $m$ deals with the fraction of advanced nodes and $N(1-m)$ is the number of normal nodes. The overall initial energy of WSN is the aggregation of heterogeneous node's energy values.

$$
\begin{gathered}
E_{\text {total }}=N(1-m) E_{o}+N m(1+a) E_{o} \\
=N E_{o}(1-m+m+a m) \\
=N E_{o}(1+a m)
\end{gathered}
$$

The two level heterogeneous WSNs contain am times more energy as compared to homogeneous WSNs.

\subsection{Three Level Heterogeneous WSN Model}

Three level heterogeneous WSNs contain three different energy levels of nodes i.e. normal, advanced and super nodes. Normal nodes contain energy of $E_{o}$, the advanced nodes of fraction $\mathrm{m}$ are having a times extra energy than normal nodes equal to $E_{o}(1+a)$ whereas, super nodes of fraction $m_{o}$ are having a factor of $\mathrm{b}$ times more energy than normal nodes so their energy is equal to $E_{o}(1+b)$. As Nis the total number of nodes in the network, then $\mathrm{Nmm}_{\mathrm{o}}$ is total number of super nodes and $N m\left(1-m_{o}\right)$ is total number of advanced nodes. The total initial energy of three level heterogeneous WSN is therefore given by:

$$
\begin{aligned}
E_{\text {total }}= & N(1-m) E_{o}+N m\left(1-m_{o}\right)(1+a) E_{o}+ \\
& N m_{o} E_{o}(1+b) \\
& E_{\text {total }}=N E_{o}\left(1+m\left(a+m_{o} b\right)\right)
\end{aligned}
$$

The three level heterogeneous WSNs contain $\left(a+m_{o} b\right)$ times more energy as compared to homogeneous WSNs.

\subsection{Multilevel Heterogeneous WSN Model}

Multi level heterogeneous WSN is a network that holds nodes of various energy levels. The initial energy of nodes is circulated over the nearby set $\left[E_{o}, E_{o}\left(1+a_{\max }\right)\right]$, where $E_{o}$ the lower is bound and $a_{\max }$ is the value of maximal energy. At first, node $S_{i}$ is equipped with initial energy of $E_{o}\left(1+a_{i}\right)$, which is $a_{i}$ times more energy than the lower bound $E_{o}$. The aggregate initial energy of multi-level heterogeneous networks is given by:

$$
E_{\text {total }}=\sum_{i=1}^{N} E_{o}\left(1+a_{i}\right)=E_{o}\left(N+\sum_{i-1}^{N} a_{i}\right)
$$

$\mathrm{CH}$ nodes devour more energy as contrasted with member nodes so after a few rounds energy level of every last one of hubs gets diverse as contrasted with one another. Therefore, heterogeneity is presented in homogeneous WSNs and the networks that hold heterogeneity are more critical than homogeneous networks.

\section{RADIO DISSIPATION MODEL}

The radio energy model depicts that 1 bit message is transmitted over a distance $d$ as in [5.6], energy exhausted is then given by:

$$
E_{T X}(1, d)=\left\{\begin{array}{c}
l E_{\text {elec }}+l \varepsilon f_{s} d^{2}, \quad d<d_{o} \\
l E_{\text {elec }}+l \varepsilon m p d^{4}, \quad d \geq d_{o}
\end{array}\right.
$$

Where, $E_{\text {elec }}$ is the energy dissipated per bit to run the transmitter or the receiver circuit. $d$ is the separation between sender and receiver. If this distance is less than threshold, free space (fs) model is used else multi path (mp) model is used .Now, total energy disseminated in the network throughout a round is given by $[5,6]$

$$
E_{\text {round }}=L\left(2 N E_{\text {elec }}+N E_{D A}+k \varepsilon_{m p} d_{\text {toBS }}^{4}+N \varepsilon_{f a} d_{\text {toCH }}^{2}(6)\right.
$$

Where, $K=$ number of clusters $E D A=$ Data aggregation cost expensed in $\mathrm{CH}$ $d$ toBS $=$ Average distance among the $\mathrm{CH}$ and $\mathrm{BS}$ $d t o C H=$ Average distance among the cluster members and the $\mathrm{CH}$

$$
\begin{array}{r}
d_{t o C H}=\frac{M}{\sqrt{2 \pi}}, d_{t o B S}=0.765 \frac{M}{2} \\
k_{\text {opt }}=\frac{\sqrt{N}}{\sqrt{2 \pi}} \sqrt{\frac{\varepsilon_{f s}}{\varepsilon_{m p}}} \frac{M}{d_{t o B S}^{2}}
\end{array}
$$

\section{OVERVIEW OF DISTRIBUTE HETEROGENOUS PROTOCOLS}

\subsection{DEEC}

DEEC is intended to manage nodes of heterogeneous WSNs. For $\mathrm{CH}$ selection, DEEC uses initial and remaining energy level of nodes. Let $n_{i}$ indicates the number of rounds to be a $\mathrm{CH}$ for node $s_{i} \cdot p_{\text {opt }} \mathrm{N}$ is the ideal number of $\mathrm{CHs}$ in the network throughout every round. A $\mathrm{CH}$ selection criterion in DEEC is focused around energy values of nodes. As in homogenous network, when nodes have equal measure of energy throughout every epoch then choosing $p_{i}=p_{\text {opt }}$ guarantees that $p_{\text {opt }} \mathrm{N} \mathrm{CHs}$ throughout every round. In WSNs, nodes with high energy are more reasonable to become $\mathrm{CH}$ than nodes with low energy but the net value of $\mathrm{CH}$ throughout every round is equivalent to $p_{o p t} \mathrm{~N}$. $\mathrm{p}_{\mathrm{i}}$ is the probability for each node $s_{i}$ to become $\mathrm{CH}$, so, node with high energy has larger value of $p_{i}$ as contrasted to the $p_{\text {opt }} . \bar{E}(r)$ depicts average energy of network during round $r$ which can be given as in [10]: 


$$
E(r)=\frac{1}{N} \sum_{i=1}^{N} E_{i}(r)
$$

Probability for $\mathrm{CH}$ selection in DEEC is given as in [10]:

$$
p_{i}=p_{\text {opt }}\left[1-\frac{\bar{E}(r)-E_{i}(r)}{\bar{E}(r)}\right]=p_{\text {opt }} \frac{E_{i}(r)}{\bar{E}(r)}
$$

In DEEC the average sum number of $\mathrm{CH}$ during every round is given as in [10]:

$\sum_{i=1}^{N} p_{i}=\sum_{i=1}^{N} p_{\text {opt }} \frac{E_{i}(r)}{\bar{E}(r)}=p_{\text {opt }} \sum_{i=1}^{N} \frac{E_{i}(r)}{\bar{E}(r)}=N p_{\text {opt }}$

$p_{i}$ is probability of every node to be a $\mathrm{CH}$ in a round. Where $\mathrm{G}$ is the set of nodes suitable to become $\mathrm{CH}$ at round $r$. If node becomes $\mathrm{CH}$ in recent rounds then it is from set $\mathrm{G}$. Throughout every round every node selects a random number between 0 and 1 . If number is minimum than threshold value as explained in equation 12 as in [10], it is appropriate to become a $\mathrm{CH}$ else not.

$$
T\left(s_{i}\right)= \begin{cases}\frac{p_{i}}{1-p_{i}\left(\operatorname{rmod} \frac{1}{p_{i}}\right)} & \text { if } s_{i} \in G \\ 0 & \text { otherwise }\end{cases}
$$

As $p_{\text {opt }}$ is reference value of average probability $p_{i}$. In homogenous networks, all nodes have same initial energy so they use $p_{\text {opt }}$ to be the reference energy for probability $p_{i}$. However in heterogeneous networks, the value of $p_{\text {opt }}$ is diverse according to the initial energy of the node. In two level heterogeneous network the value of $p_{\text {opt }}$ is given by as in[10]:

$$
p_{a d v}=\frac{p_{o p t}}{1+a m}, p_{n r m}=\frac{p_{\text {opt }}(1+a m)}{(1+a m)}
$$

Then use the above $p_{a d v}$ and $p_{n r m}$ in its place of $p_{o p t}$ in equation 10 for two level heterogeneous networks as assumed in [10]:

$$
p i=\left\{\begin{array}{cc}
\frac{p_{\text {opt }} E_{i}(r)}{(1+a m) \bar{E}(r)} & \text { if } s_{i} \text { is the normal node } \\
\frac{p_{\text {opt }}(1+a) E_{i}(r)}{(1+a m) \bar{E}(r)} & \text { if } s_{i} \text { is the advanced node }
\end{array}\right.
$$

Above model can also be extended to multi level heterogeneous network given below as in [10]:

$$
p_{\text {multi }}=\frac{p_{o p t} N\left(1+a_{i}\right)}{\left(N+\sum_{i=1}^{N} a_{i}\right)}
$$

Above $p_{\text {multi }}$ in equation 10 instead of $p_{\text {opt }}$ to get $p_{i}$ for heterogeneous node. $p_{i}$ for the multilevel heterogeneous network is specified by as in [10]:

$$
p_{\text {multi }}=\frac{p_{\text {opt }} N\left(1+a_{i}\right)}{\left(N+\sum_{i=1}^{N} a_{i}\right)}
$$

In DEEC we approximate average energy $E(r)$ of the network for any round $r$ as in [10]:

$$
\bar{E}(r)=\frac{1}{N} E_{\text {total }}\left(1-\frac{r}{R}\right)
$$

$R$ denotes total rounds of network lifetime and is estimated as follows:

$$
R=\frac{E_{\text {total }}}{E_{\text {round }}}
$$

Etotal is total energy of the network where Eround is energy expenditure during each round.

\subsection{DDEEC}

DDEEC utilizes same strategy for estimation of average energy in the network and $\mathrm{CH}$ selection algorithm focused around residual energy as applied in DEEC. Distinction between DDEEC and DEEC is focused in expression that characterizes probability for normal and advanced nodes to become a $\mathrm{CH}$ [11] as given in equation 14 . We find that nodes with more residual energy at round $r$ are more reasonable to become $\mathrm{CH}$, so, in this method node having higher energy values or advanced nodes will become $\mathrm{CH}$ more often as compared to the nodes with lower energy or normal nodes. At a certain point in the network where advanced nodes containing equal residual energy same as normal nodes. Although, after this point DEEC continues to punish the advanced nodes so this is not ideal way for energy allocation as by doing so, advanced nodes are constantly a $\mathrm{CH}$ and they die more quickly than normal nodes. To prevent this unbalanced case, DDEEC makes few improvements in equation 14 to keep advanced nodes from being penalized over and again. DEEC introduces threshold residual energy as in [11] and given below:

$$
T h_{R E V}=E_{o}\left(1+\frac{a E_{d i s N N}}{E_{d i s N N}-E_{d i s A N}}\right)
$$

At the point when energy level of advanced and normal nodes falls down to the limit of threshold residual energy then both kind of nodes use similar probability to become cluster head. Therefore, $\mathrm{CH}$ selection is balanced and more proficient. Threshold residual energy $T h$ is given as in [11] and given below:

$$
T h_{R E V}=\left(\frac{7}{10}\right) E_{o}
$$

Average probability $p_{i}$ for $\mathrm{CH}$ selection used in DDEEC is as follows as in [11]:

$p_{i}$

$$
= \begin{cases}\frac{p_{o p t} E_{i}(r)}{(1+a m) \bar{E}(r)} & \text { for Nml nodes, } E_{i}>T h_{R E V} \\ \frac{(1+a) p_{o p t} E_{i}(r)}{(1+a m) \bar{E}(r)} & \text { for Adv nodes, } E_{i}>T h_{R E V} \\ c \frac{(1+a) p_{o p t} E_{i}(r)}{(1+a m) \bar{E}(r)} & \text { for Avd, Nml nodes, } E_{i} \leq T h_{R E V}\end{cases}
$$

\subsection{EDEEC}

EDEEC utilizes idea of three level heterogeneous networks as explained previously. It holds three types of nodes normal, advanced and super nodes focused on initial energy. $p_{i}$ is probability used for $\mathrm{CH}$ selection and $p_{\text {opt }}$ is reference for $p_{i}$ EDEEC utilizes diverse $p_{\text {opt }}$ values for normal, advanced and super nodes, so, value of $p_{i}$ in EDEEC is as follows as in [12]: 


$$
p_{i=}\left\{\begin{array}{l}
\frac{p_{o p t} E_{i}(r)}{\left(1+m\left(a+m_{o} b\right)\right) \bar{E}(r)} \quad \text { if } s_{i} \text { is the normal node } \\
\frac{(1+a) p_{o p t} E_{i}(r)}{\left(1+m\left(a+m_{o} b\right)\right) \bar{E}(r)} \quad \text { if } s_{i} \text { is the advance node } \\
\frac{(1+b) p_{o p t} E_{i}(r)}{\left(1+m\left(a+m_{o} b\right)\right) \bar{E}(r)} \quad \text { if } s_{i} \text { is the super node }
\end{array}\right.
$$

Threshold for $\mathrm{CH}$ selection for all three types of node is asfollows as in [12]:

$$
T\left(s_{i}\right)=\left\{\begin{array}{l}
\frac{p_{i}}{1-p_{i}\left(\operatorname{rmod} \frac{1}{p_{i}}\right)} \text { if } p_{i} \in G^{\prime} \\
\frac{p_{i}}{1-p_{i}\left(\operatorname{rmod} \frac{1}{p_{i}}\right)} \text { if } p_{i} \in G^{\prime \prime} \\
\frac{p_{i}}{1-p_{i}\left(\operatorname{rmod} \frac{1}{p_{i}}\right)} \text { if } p_{i} \in G^{\prime \prime \prime} \\
0 \quad \text { otherwise }
\end{array}\right.
$$

\subsection{TDEEC}

TDEEC utilizes similar technique for $\mathrm{CH}$ selection and average energy approximation as suggested in DEEC. In every round, nodes make a decision whether to become a $\mathrm{CH}$ or not by selecting a random value between 0 and 1 . If value is smaller than threshold Ts as illustrated in equation (24) then nodes choose to be a $\mathrm{CH}$ for the specified round. In TDEEC, threshold value is balanced and based upon that value a node chooses whether to turn into a $\mathrm{CH}$ or not by presenting residual energy and average energy of that round regarding to ideal number of CHs. [13]. Threshold value suggested by TDEEC is specified as follows as in [13]:

$$
T(s)=\left\{\frac{p}{1-p\left(\operatorname{rmod} \frac{1}{p}\right)} * \frac{\text { residual energy of a node } * k_{\text {opt }}}{\text { average energyof the network }}\right.
$$

\subsection{EDDEEC}

In this paper protocol implements the thought of probabilities for $\mathrm{CH}$ choice based on initial, remaining energy level of the nodes and average energy of network. This protocol protects the super and advance nodes from over penalized, because after some rounds some super and advance nodes have same residual energy level as normal nodes due to repeatedly $\mathrm{CH}$ selection. EDDEEC estimates probabilities of normal, advance and super nodes. These changes are focused on absolute residual energy level $T_{\text {absolute }}$, which is the value in which advance and super nodes containing similar energy level as that of normal nodes. The thought states that under Tabsolute all normal, advance and super nodes have equal probability for $\mathrm{CH}$ selection. The probability for three types of nodes given by EDEEC is specified below

$p_{i}=\left\{\begin{array}{l}\frac{p_{\text {opt }} E_{i}(r)}{\left(1+m\left(a+m_{o} b\right)\right) \bar{E}(r)} \quad \text { if } s_{i} \text { is the normal node } \\ \frac{(1+a) p_{o p t} E_{i}(r)}{\left(1+m\left(a+m_{o} b\right)\right) \bar{E}(r)} \quad \text { if } s_{i} \text { is the advance node (25) } \\ \frac{(1+b) p_{o p t} E_{i}(r)}{\left(1+m\left(a+m_{o} b\right)\right) \bar{E}(r)} \quad \text { if } s_{i} \text { is the super node }\end{array}\right.$
Our suggested probabilities for $\mathrm{CH}$ selection in EDDEEC are given as follows:

$$
\begin{aligned}
& p_{i} \\
& =\left\{\begin{array}{cc}
\frac{p_{\text {opt }} E_{i}(r)}{\left(1+m\left(a+m_{o} b\right)\right) \bar{E}(r)} & \text { for } N_{m l} \text { nodes } \\
\frac{(1+a) p_{o p t} E_{i}(r)}{\left(1+m\left(a+m_{o} b\right)\right) \bar{E}(r)} & \text { if } E_{i}(r)>T_{\text {absolute }} \\
\frac{(1+b) p_{o p t} E_{i}(r)}{\left(1+m\left(a+m_{o} b\right)\right) \bar{E}(r)} & \text { if } E_{i}(r)>T_{\text {absolute }} \\
\text { ifor Sup nodes } \\
\frac{(1+b) p_{o p t} E_{i}(r)}{\left(1+m\left(a+m_{o} b\right)\right) \bar{E}(r)} \text { for } E_{i}(r)>T_{\text {absolute }} \\
& \text { if } E_{i}(r) \leq T_{\text {absolute }} \text { Adv, Sup nodes }
\end{array}\right.
\end{aligned}
$$

The value of absolute residual energy level,Tabsolute, is written as:

$$
T_{\text {absolute }} Z E_{o}
$$

where, $z Q(0,1)$. If $z=0$ then we have conventional EDEEC. In reality, advanced and super nodes may have not been a $\mathrm{CH}$ in rounds $r$, it is also probable that some of them become $\mathrm{CH}$ and similar is the case with the normal nodes. So, exact value of $z$ is not certain.

\subsection{HDEEC}

Hybrid-DEEC (H-DEEC), a chain and cluster based (hybrid) circulated method for proficient energy consumption in WSNs. In H-DEEC, selected Cluster Heads (CHs) communicate the Base Station (BS) from beta selected nodes, by utilizing multihopping. We logically partition the network into two segments, based on the residual energy of nodes. The normal nodes having more initial and residual energy will be more feasible to be $\mathrm{CHs}$ than the nodes with lesser energy. H-DEEC is focused around DEEC method for heterogeneous networks, in which all nodes utilize initial and residual energy level for cluster-head selection. Every node in the network has the details of every other fellow node. The HDEEC method is combination of two scenarios; clustering and chain construction.

Clustering: As specified above, fraction of the network consist of normal node will follow the clustering scenario as done in DEEC and as illustrated in Figure 1. All nodes in the network are alert of their fellow nodes energy level and location. Normal node will track the similar approach in terms of calculating average energy of the network, and $\mathrm{CH}$ selection algorithm, probability of each node to become $\mathrm{CH}$ is based on residual energy. For network of $\mathrm{N}$ nodes and an extra energy factor, and it is computed as:

$$
p_{i}=\frac{p_{o p t} N(1+a) E_{i}(r)}{\left(N+\sum_{i=1}^{N} a_{i}\right) \bar{E}(r)}
$$

Where $p_{\text {opt }}$ is the reference value of the average probability $p_{i}$, which decide the rotating epoch. $\bar{E}(\mathrm{r})$ is average energy of $r t h$ round and $E_{i}$ is the residual energy of node $s_{i}$ at round $r$. We can approximate average energy at $r$ th round as given below:

$$
\bar{E}(r)=\frac{1}{N} E_{\text {total }}\left(1-\frac{r}{R}\right)
$$


$R$ depicts the number of rounds a network will be alive and is approximated as:

$$
R=\frac{E_{\text {total }}}{E_{\text {round }}}
$$

Chain Construction: Chain construction of beta nodes in our proposed situation is done based on traditional chain routing method PEGASIS. It preserves a considerable amount of energy as contrasted with the other routing protocols, because of its enhancement in delivery of data. Beta nodes will be attached by subsequent greedy algorithm. BS begins the chain constructing procedure by marking the extreme node. Farthest node finds its nearest neighbour and so on. Leader node of the chain is chosen as the beta node with least distance from BS. As chain turn around in each round based on the energy and beta node chain all the time will be redirected in the direction of the BS.

\section{PERFORMANCE CRITERIA}

Performance parameters are utilized for evaluation of clustering protocols for heterogeneous WSNs are lifetime of heterogeneous WSNs, number of nodes alive throughout rounds and data packets transmitted to BS.

Lifetime is a parameter used to show node of each type has not up till now spent its total energy.

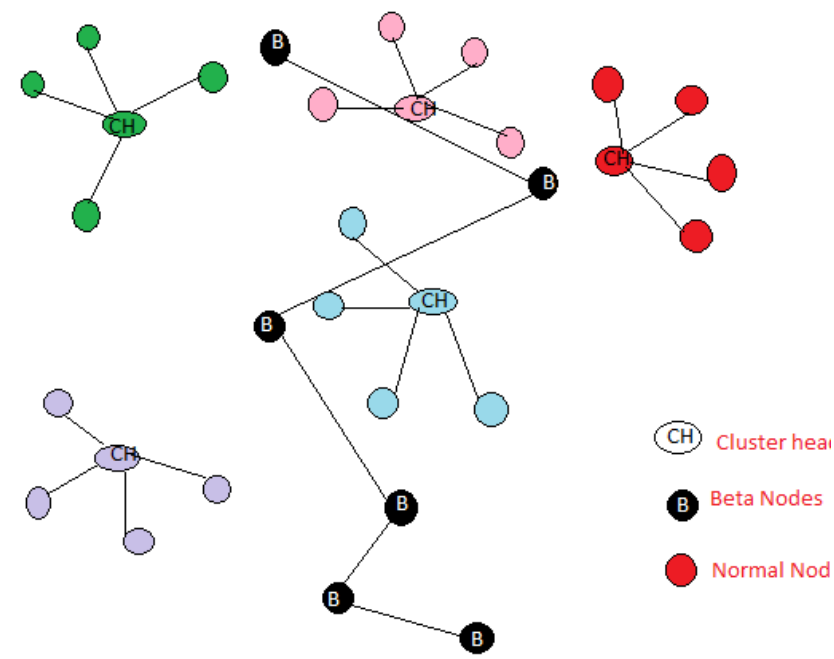

Fig 1.Network Model of HDEEC

Number of nodes alive is a parameter which defines number of alive nodes through every round.

Data packets transmitted to the Base Station is the metric which describes how many packets are received by Base Station for every round.

These parameters show stability period, instability period, energy consumption, data sent to the BS, and data received by BS and network lifetime of WSNs.

Stability period is period from start of network until the death of first node whereas; instability period is period from the death of first node until last one.

\section{SIMULATIONS AND DISCUSSIONS}

In this section, we simulate different clustering protocols in heterogeneous WSN using MATLAB and for simulations we use 100 nodes randomly placed in a field of dimension $100 \mathrm{~m} \times 100 \mathrm{~m}$. For ease, we suppose all nodes are either fixed or micro-mobile and avoid energy loss because of signal collision and interference between signals of different nodes that are due to dynamic random channel conditions.

Table 1: WSNs Set-up
\begin{tabular}{|l|l|}
\hline Parameter & Value \\
\hline Area(x,y) & 100,100 \\
\hline Base station(x,y) & 50,50 or 50,150 \\
\hline Nodes(n) & 100 \\
\hline Probability(p) & 0.1 \\
\hline Initial Energy & 0.1 \\
\hline transmiter_energy & $50 * 10^{-9}$ \\
\hline receiver_energy & $50 * 10^{-9}$ \\
\hline Free space(amplifier) & $10 * 10^{-13}$ \\
\hline Multipath(amplifier) & $0.0013 * 10^{-13}$ \\
\hline Effective Data aggregation & $5 * 10^{-9}$ \\
\hline Maximum lifetime & 2500 \\
\hline Data packet Size & 4000 \\
\hline m (fraction of advanced nodes) & 0.3 \\
\hline $\begin{array}{l}\text { a (energy factor between normal } \\
\text { and advanced nodes) }\end{array}$ & 1.5 \\
\hline$m_{o}$ fraction of super nodes & 0.3 \\
\hline
\end{tabular}

In this scenario, we are taking into account that, BS is located at centre of the network field. We simulate DEEC, DDEEC, EDEEC and TDEEC, EDDEEC and H-DEEC for three-level and multi-level heterogeneous WSNs. Scenarios depicts values for number of nodes dead in first, tenth and last rounds also values for the packets transmitted to $\mathrm{BS}$ by $\mathrm{CH}$ and values for packets transmitted to $\mathrm{CH}$ by nodes at distinct values of parameters $\mathrm{m}, \mathrm{m}_{\mathrm{o}}$, $\mathrm{a}$ and $\mathrm{b}$. These values are observed for DEEC, H-DEEC, DDEEC, EDEEC, EDDEEC and TDEEC.

In heterogeneous WSN, we utilized radio parameters stated in Table 1 for distinctive protocols placed in WSN and estimate the performance for three level heterogeneous WSNs. Parameter $\mathrm{m}$ refers to fraction of advanced nodes having additional amount of energy ain network whereas, $m_{o}$ is a factor that refers to fraction of super nodes having additional amount of energy $b$ in the network.

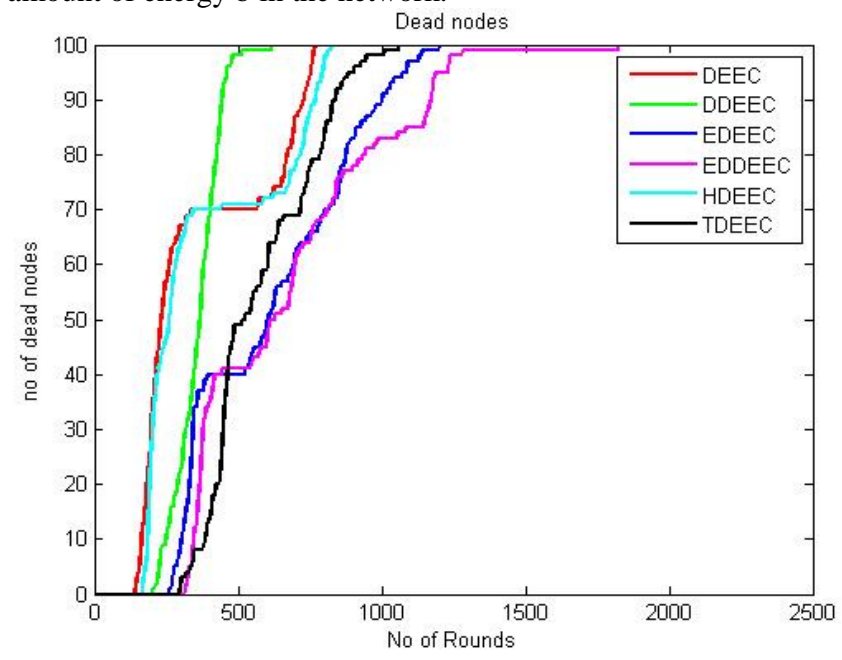

Fig. 2 Nodes dead during rounds 

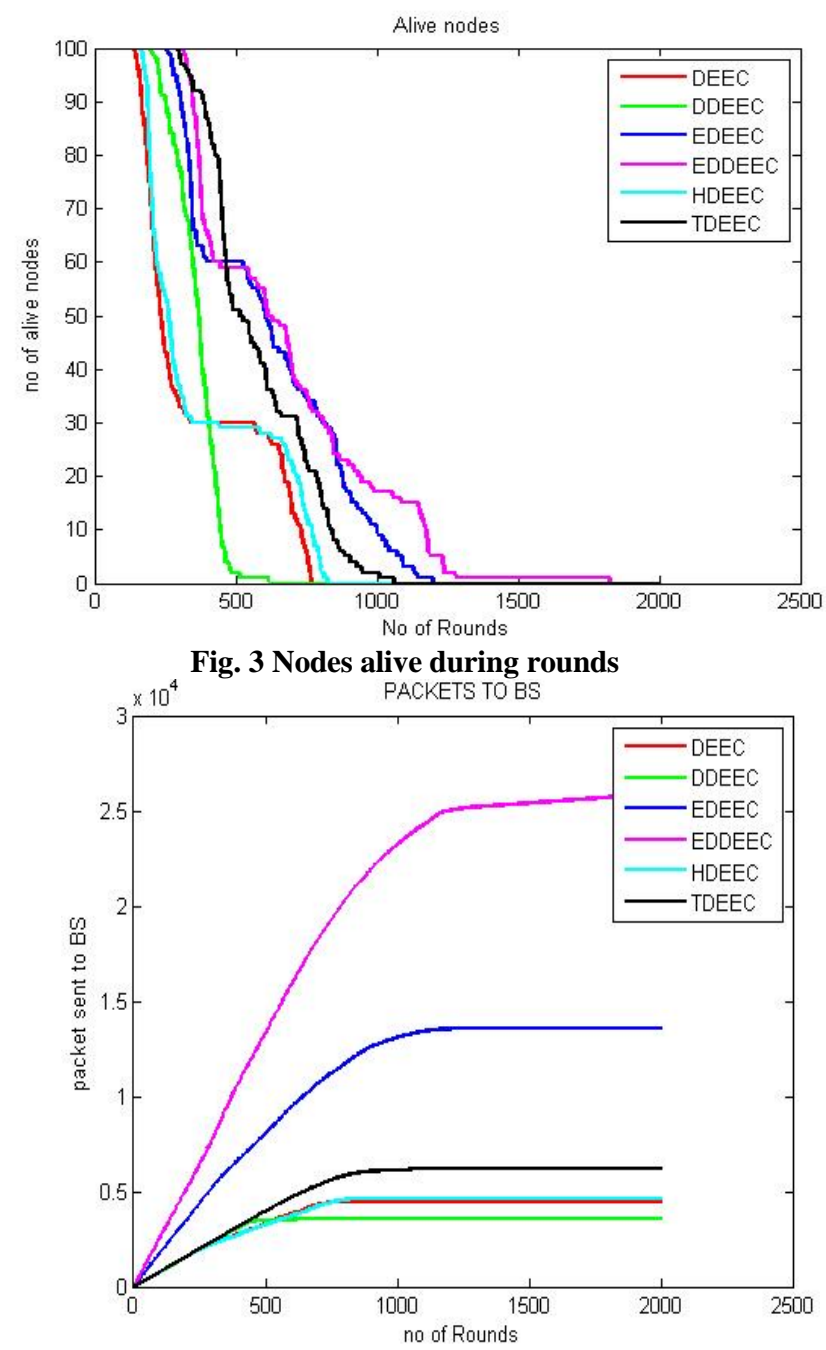

Fig. 4 Packets sent to $B S$

For the case of a network containing $\mathrm{m}=0.3$ fraction of advanced nodes having $\mathrm{a}=1.5$ times more energy and $m o=0.3$ fraction of super nodes containing $b=3$ times more energy than normal nodes. From Fig. 2 and 3, we examine that first node for DEEC, DDEEC, EDEEC TDEEC, HDEEC and EDDEEC dies at $140,201,258,297,166$ and 315 rounds respectively. Tenth node dies at 164, 249, 303, 384, 184 and 344 rounds respectively.

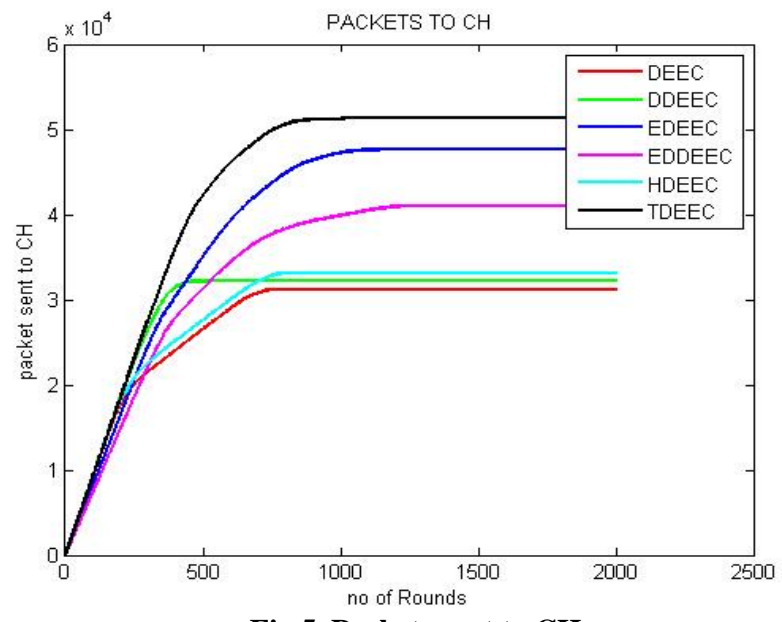

Fig 5. Packets sent to $\mathrm{CH}$
All nodes are dead at 769, 617, 1201, 1058, 825 and 1822 rounds respectively. It is obvious from the results of all protocols that in terms of stability period, EDDEEC performs best of all; TDEEC performs better than DEEC, EDEEC, DDEEC, and HDEEC but has less performance than EDDEEC. HDEEC only performs well as compared to DEEC and DEEC has least performance than all the protocols. Though, instability period of EDDEEC is best of all. EDEEC and TDEEC perform better than HDEEC, DDEEC and DEEC. The number of nodes alive in EDDEEC is quite larger than EDEEC because in EDDEEC the formula of threshold used by nodes for $\mathrm{CH}$ election is modified by including residual and average energy of that round. So nodes containing high energy will become CHs. DDEEC has less performance among all. Similarly, by examining results of Fig. 4, packets sent to the BS by DEEC, DDEEC, EDEEC, TDEEC, HDEEC and EDDEEC have their values at 4470, 3525, 13551, 6143, 4609 and 25721.

Now we observe that packets transmitted to BS for DEEC and DDEEC is approximately equal while, the packets transmitted to BS for EDEEC and TDEEC are approximately the equal as the probability equations for normal, advanced and super nodes is similar in both of them. Packet transmission in EDDEEC is maximum among all. Now approaching to the $\mathrm{CHs}$, the packets transmitted to $\mathrm{CHs}$ increase throughout the beginning of the network and slowly decrease downwards the end because the nodes vanishing concurrently.

Now considering second case in which the parameters change to $\mathrm{a}=1.5, \mathrm{~b}=3, \mathrm{~m}=0.3$ and $\mathrm{mo}=0.3$, area $(\mathrm{x}, \mathrm{y})=50,150$. Fig. 6 shows that first node for DEEC, DDEEC, EDEEC, TDEEC, HDEEC and EDDEEC dies of each protocol at 111, 185, 158, 228,145 and147 rounds respectively. Tenth node dies at 132, 205, 215,369,180 and 203 rounds respectively.

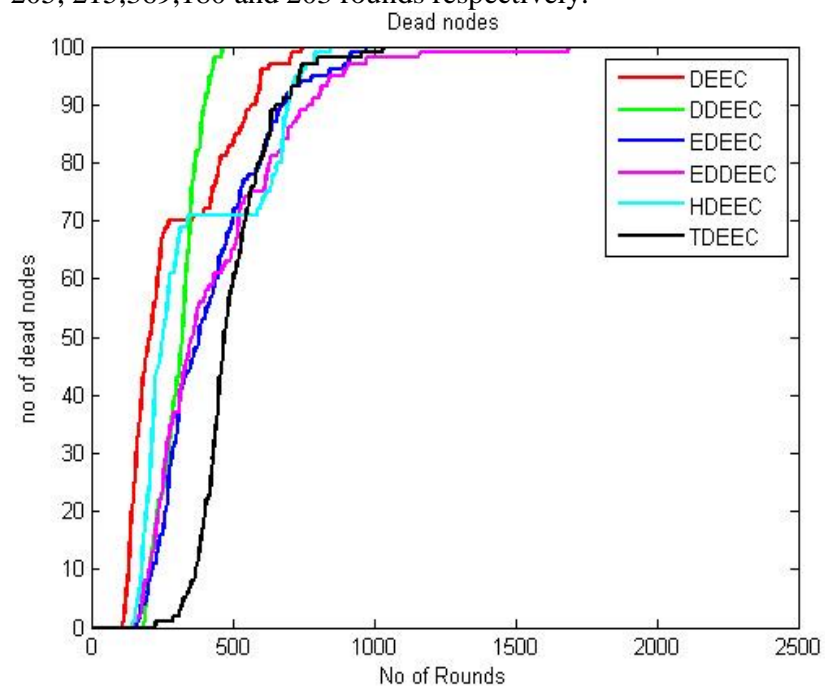

Fig. 6. Nodes dead during round $(50,150)$ 


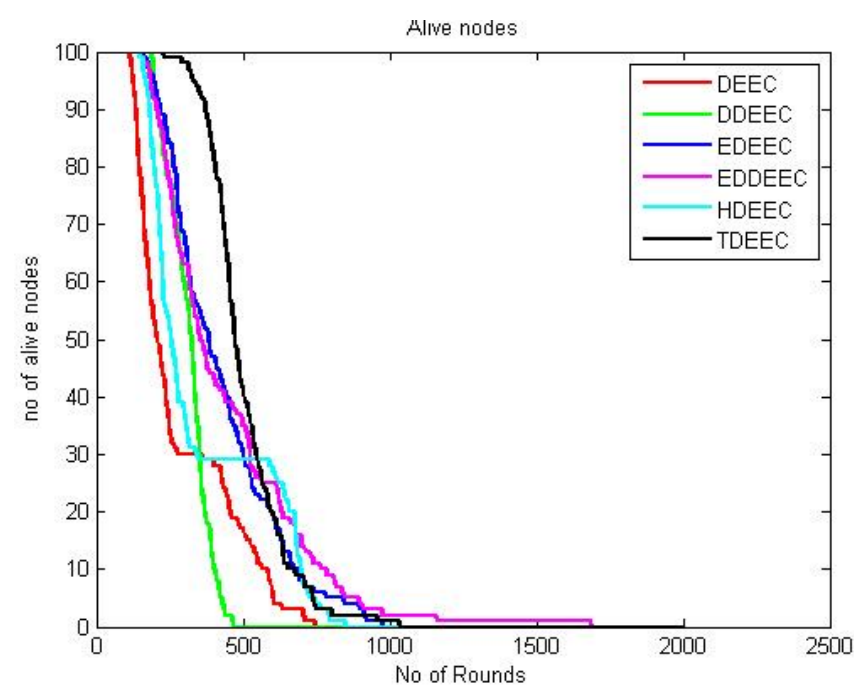

Fig 7 Nodes alive during rounds

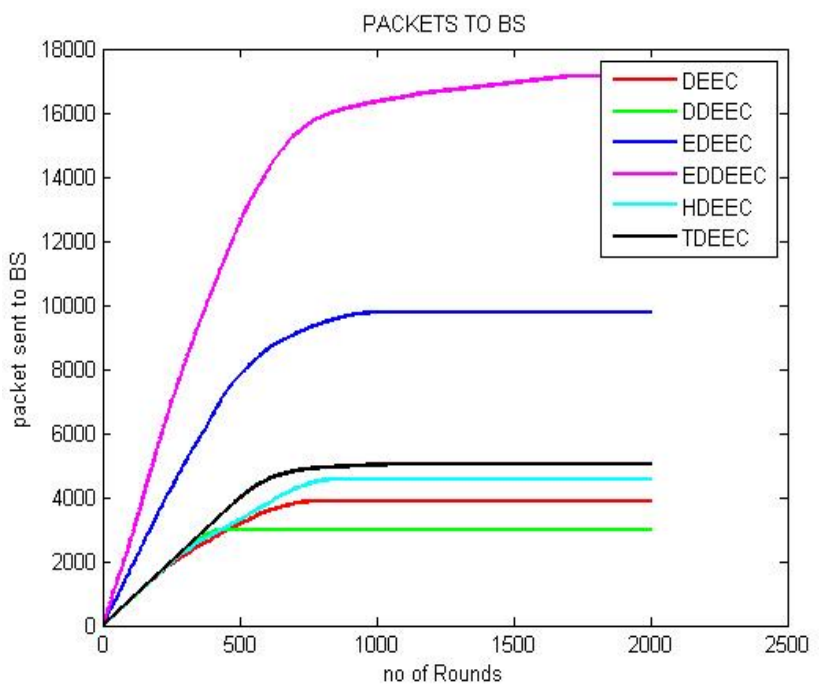

Fig.8 Packets to BS 50,150

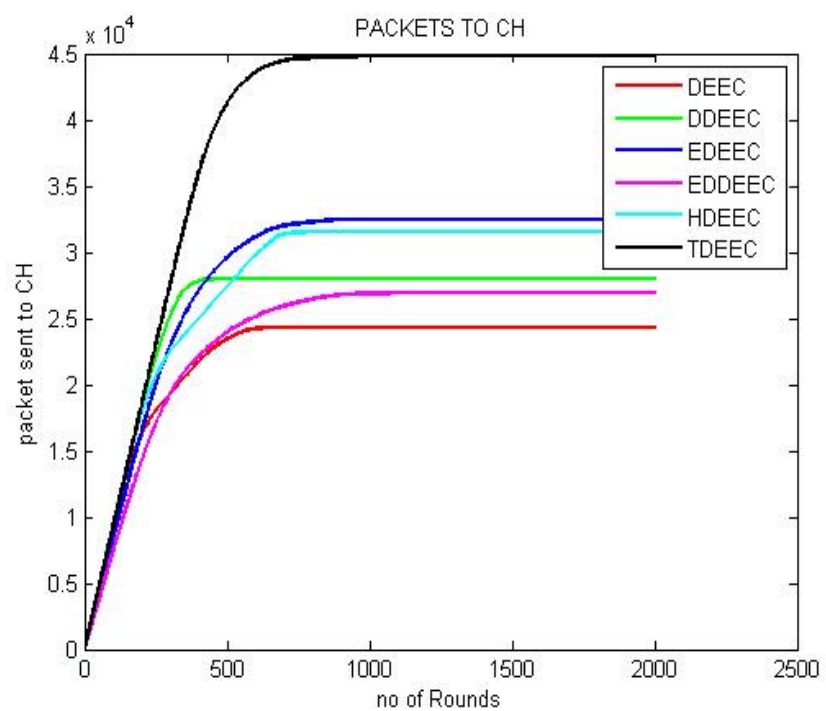

Fig. 9. Packets sent to $\mathbf{C H}$

All nodes are dead at 746, 463, 972, 1034, 846 and1689 rounds. Alive in first, tenth and all rounds is accurately the flip to the graph for number of nodes dead and is shown in Fig. 7. Result of Fig. 8 shows that packets sent to BS by DEEC, DDEEC, EDEEC, TDEEC, HDEEC and EDDEEC are $3862,2987,9763,5018,4572$ and 17128 respectively. As we see that by decreasing the values of parameters, EDDEEC still performs best among the six protocols. EDEEC performs better than TDEEC. HDEEC performs better than DEEC and DDEEC whereas, DDEEC performs worst.

\section{CONCLUSION AND FUTURE WORK}

This paper has evaluated and compares the well-known heterogeneous WSNs energy efficient protocols i.e. DEEC variants. The comparison has shown that the EDDEEC has quite effective results over the other DEEC variants. Although EDDEEC has shown quite significant results over existing WSNs protocols but it has neglected the use of waiting time of node to become CHs. So may some nodes will not become $\mathrm{CHs}$ for a long time even they have more confidence to become $\mathrm{CHs}$.

So to overcome this problem in near future we will use minimum allowed distance $(\mathrm{MDCH})$ and waiting nodes between two $\mathrm{CHs}$ to divide the sensor field among clusters in the most efficient way. MDCH will have ability to overcome the problem of the too small and too high cluster heads.

\section{REFERENCES}

[1] I.F. Akyildiz, W. Su, Y. Sankara subramaniam, E. Cayirci, "Wireless sensor networks: a survey, Computer Networks", 38 (4) (2002) 393-422.

[2] P. Krishna, N.H. Vaidya, M. Chatterjee, D. Pradhan, “A cluster-based approach for routing in dynamic networks, ACM SIGCOMM Computer Communication Review", 27 (2) (1997) 49-65.

[3] B. McDonald, T. Znati, "Design and performance of a distributed dynamic clustering algorithm for Ad-Hoc networks", in: Proceedings of the Annual Simulation Symposium, 2001

[4] V. Mhatre, C. Rosenberg, D. Kofman, R. Mazumdar, N. Shroff, "Design of surveillance sensor grids with a lifetime constraint", in: 1st European Workshop on Wireless Sensor Networks (EWSN), Berlin, January 2004.

[5] W.R. Heinzelman, A.P. Chandrakasan, H. Balakrishnan, "Energy efficient communication protocol for wireless micro sensor networks", in: Proceedings of the 33rd Hawaii International Conference on System Sciences(HICSS-33), January 2000.

[6] W.R. Heinzelman, A.P. Chandrakasan, H. Balakrishnan, "An application specific protocol architecture for wireless micro sensor networks", IEEE Transactions on Wireless Communications 1 (4) (2002) 660-670.

[7] S. Lindsey, C.S. Raghavenda, PEGASIS: "power efficient gathering in sensor information systems", in: Proceeding of the IEEE Aerospace Conference, Big Sky, Montana, March 2002.

[8] O. Younis, S. Fahmy, HEED: "A hybrid, energy-efficient, distributed clustering approach for ad hoc sensor networks", IEEE Transactions on Mobile Computing 3 (4) (2004) 660-669.

[9] G. Smaragdakis, I. Matta, A. Bestavros, "SEP: A Stable Election Protocol for clustered heterogeneous wireless sensor network", in: Second International Workshop on Sensor and Actor Network Protocols and Applications (SANPA 2004), 2004. 
[10] L. Qing, Q. Zhu, M. Wang, "Design of a distributed energy-efficient clustering algorithm for heterogeneous wireless sensor network", ELSEVIER, Computer Communications 29, 2006, pp 2230- 2237.

[11] Elbhiri, B., Saadane, R., El Fkihi, S., Aboutajdine, D. "Developed Distributed Energy-Efficient Clustering (DDEEC) for heterogeneous wireless sensor networks", in: 5th International Symposium on I/V Communications and Mobile Network (ISVC), 2010.

[12] Parul Saini, Ajay. K. Sharma, "E-DEEC- Enhanced Distributed Energy Efficient Clustering Scheme for heterogeneous WSN", in: $2010 \quad 1^{\text {st International }}$ Conference on Parallel, Distributed and Grid Computing (PDGC - 2010).
[13] Parul Saini, Ajay. K. Sharma, "Energy Efficient Scheme for Clustering Protocol Prolonging the Lifetime of Heterogeneous Wireless Sensor Networks", International Journal of Computer Applications (0975 8887), September 2010.

[14] M. Y. Khan, N. Javaid, M. A. Khan,'Hybrid DEEC: Towards Efficient Energy Utilization in Wireless Sensor Networks", Published in World Applied Science Journal (ISI Indexed), 2013.

[15] N. Javaid, T. N. Qureshi, A. H. Khan, A. Iqbal, E. Akhtar, M. Ishfaq, "EDDEEC: Enhanced Developed Distributed Energy-Efficient Clustering for Heterogeneous Wireless Sensor Networks", ELSEVIER 4th International Conference on Ambient Systems, Networks \& Technologies (ANT) 2013. 